\title{
Research and Application Progress of Social Media in China's Tourism
}

\author{
Chunyan Wang \\ College of Business Administration, Jilin Engineering Normal University, Changchun, China \\ 541012329@qq.com
}

Keywords: Social media; Tourism destination; Marketing; Literature review

\begin{abstract}
The use of social media by modern tourists is increasing, especially in the aspect of information searching and decision-making about tourist destination. The tourist destination area carries on the traveling marketing and the visitor interaction by using the social media to promote the destination and propagate the traveling products. In this paper, it collects the literature related to social media in China's tourism study, generalizes and comments the research status of social media in China's tourism, summarizes the research achievements of Chinese scholars, and provides some personal views on the future direction of research. The study shows that social media research in tourism is still in its infancy in China. In the future, we should have a more comprehensive understanding and in-depth research on social media, so that it can be used in tourism management and marketing activities, and then generates profound influence on the entire tourism industry.
\end{abstract}

\section{Introduction}

Modern tourism industry is largely dependent on an electronic network. Destination's reputation, consumer's opinions, information dissemination and oral propaganda are using social media, so the interpretation of the role of social media has become one of the main tasks of the modern tourism research [1]. Social media refers to "computer-mediated tools that allow people, companies and other organizations to create, share, or exchange information, career interests, ideas, and pictures/videos in virtual communities and network [2]. Social media is an important network tools, including social networking sites, word-of-mouth reviews websites, content sharing sites, Wikipedia websites, forums and local social media, etc.. In social media, the behavior of sharing and exchanging information using media, such as through text, pictures, video and audio and other media, gradually become a form of social interaction. In recent years, the rapid development of China's social media, as of December 2015, in China micro-blog's active population has reached 236 million monthly. According to eMarketer's latest report, in 2015 China's users spend 6 hours and 8 minutes in all kind of media every day, and the number of Wechat's active account at home and abroad monthly has reached 697 million[3].

Social media is a new and emerging field of tourism research, which is different from the traditional transmission mode of travel information. Stratified users by social media for achieving precision marketing has become a mature marketing tool, and social media has been seen as an important tool for upgrading the tourism industry by many countries [4]. In this paper, the use of social media in tourism as the object, it summarizes the relevant information and analyzes its progress, looking for research trends and differences, extracts useful inspiration for development of tourism.

\section{Data Acquisition}

Using titles and themes as search condition, we made a retrieval in CNKI on December $30^{\text {th }}, 2015$, combined the keyword "social media" with "tourism", or matched "social networking", "Web 2.0", "user generated content (UGC)" with "tourists", "travel" and "hospitality", in which similar words can be interchanged, a total of 458 articles. To ensure the timeliness and scientificalness, we searched articles again using above method on May $1^{\text {st }}, 2015$. And the latest relevant research achievements, which are collected by main databases, are drawn into the scope of statistics. After deleting the 
repetition and low correlation, a total of 497documents are retained. After a review of the abstracts and keywords of literatures, eventually the sample data are reconstructed using Excel, and the indicators include author, title, published time, journal, abstract and keywords etc.. On the basis of the review summary, we select those key articles to read.

\section{Statistical Analysis}

Published Time. Research on social media related tourism in China has a short history. Chinese scholars began to introduce the concept of social media in 2006 and pointed out the "tourism 2.0" era is coming (a total of 2 papers, which were both published in the Tourism Tribune). From 2007 to 2010, the number of articles published is very small. After 2010, the number of articles published rapidly increased and the research focus gradually shifted to interactive with visitors by using social media, rich tourist experience, tourism marketing and services, etc.. Its research peak appeared from 2014 to 2015 (shown in Fig. 1).

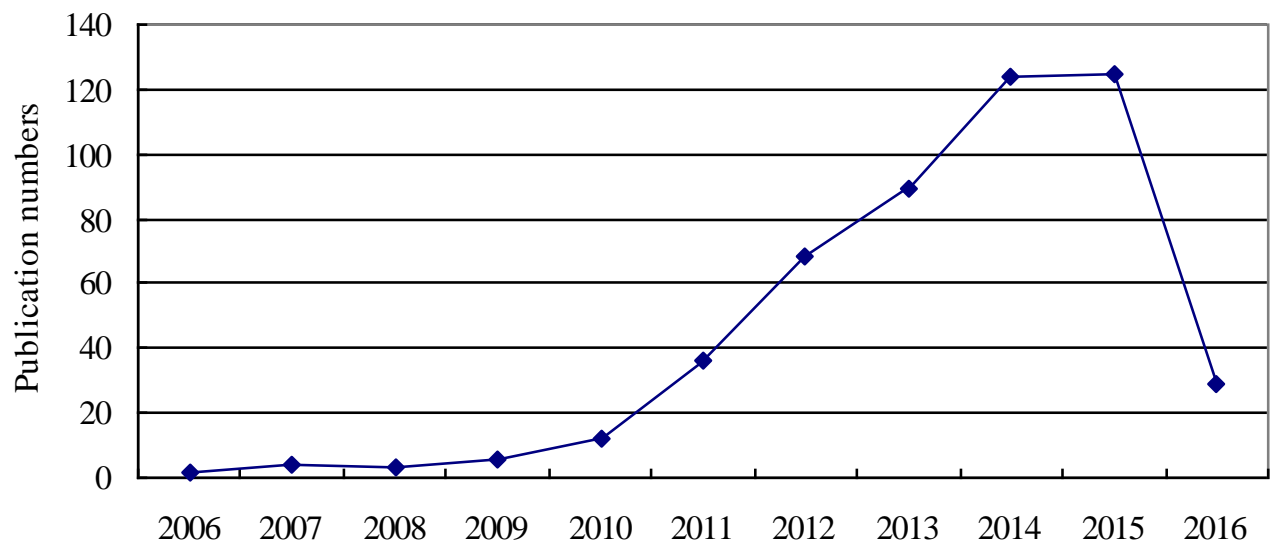

Figure 1. Publication numbers of papers by year (from Jan. 2006 to May 2016)

Sources of Literatures. The distribution of research results is more concentrated, the largest proportion for academic journals (335 papers, accounting for 67.4\%), secondly for Mater and Doctoral Dissertations (118 papers, accounting for $23.8 \%$ ), thirdly for newspapers (34 papers, accounting for $6.8 \%$ ), finally for the conference papers (10 papers, accounting for $2.0 \%$ ). In Chinese academic journals, some professional core periodicals pay attention to the social media, such as "Tourism Tribune". At that time, many scholars had predicted that the Web2.0 will bring a revolutionary change in tourism. Since 2007, some postgraduates of tourism management selected social media as their graduation topics in Chinese universities. As of December 2015, a total of 118 related mater and doctoral dissertations about social media in tourism had been completed from 42 universities in China. From the geographical distribution, these 42 universities are located in Beijing, Tianjin, Shanghai, Zhejiang, Guangdong and other developed areas. In the statistical results of the conference papers, there are only 10 Chinese Conference papers. Perhaps it is limited to the stage of development of tourist information, Chinese scholars is a lack of achievements in the field of international exchange.

High Frequency Keywords. A total of 158 key words were analyzed from 53 Chinese literatures. Through the same process, the final outcome: high frequency keywords followed by travel website/ network platform (including network technology, such as RSS4, network geographic information system), marketing (including network marketing, destination marketing), tourist behavior, destination, cultural creativity (shown in Fig. 2) as well as other classes. Statistical data show that the research direction of domestic scholars mainly focus on the platform construction of social media in tourism, the tourism marketing by social media, the tourists' behavior, and the tourism destination management. In the term of research focus, Chinese scholars pay more attention to the practical application and concentrate some field, such as marketing, destination management and tourist behavior. But they are 
not involved in some aspects of social media on its use subject and community about cultural, ethical and legal issues.

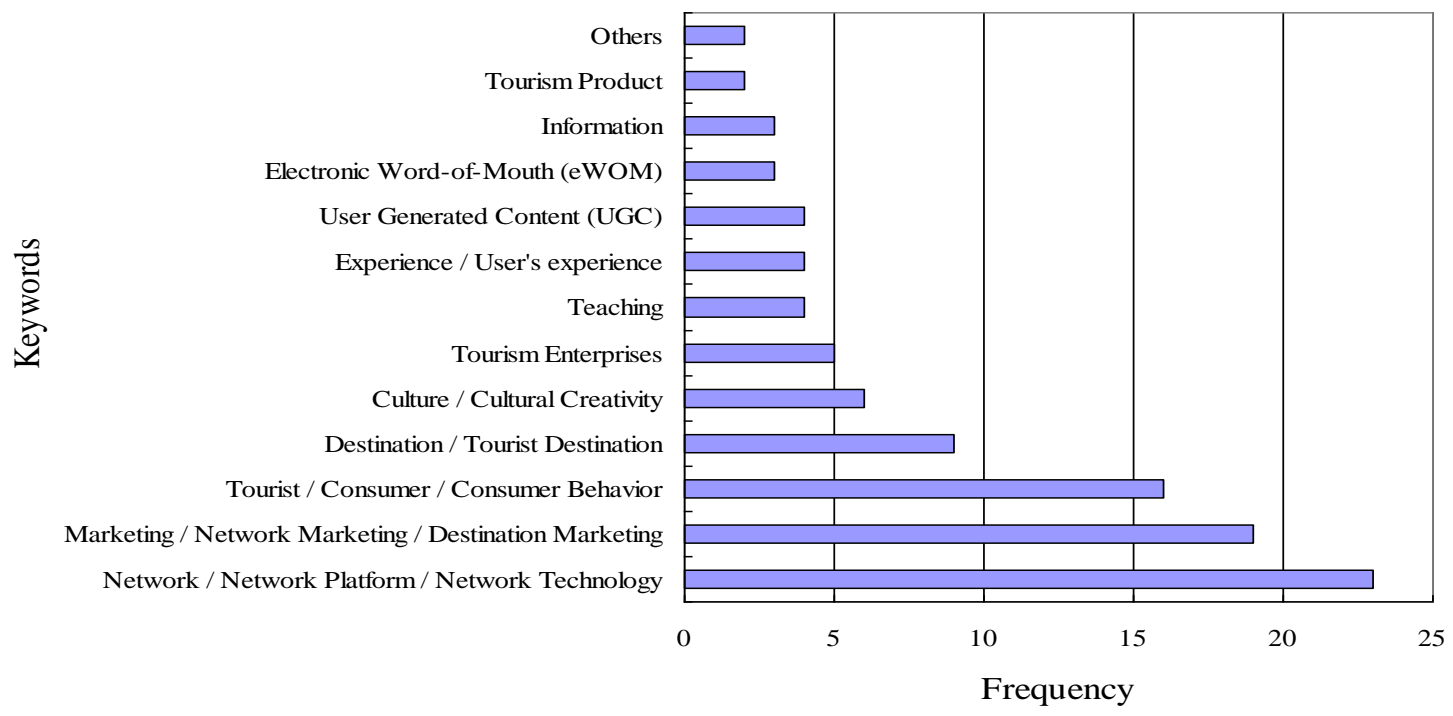

Figure 2. Presence frequencies of keywords in Chinese journal papers

Research Methods. Scholars in the study of tourism social media used a variety of research methods flexibly and effectively, including quantitative analysis and qualitative analysis. Online survey has become the main method to collect data, especially when the research object is social media users or tourists [5]. Many studies have used case studies or empirical research methods. These methods mainly focus on the definition and analysis of social networks, the ability of creating knowledge, the practical application significance of social network in the tourism [6], user (tourists) generated content [7], and the interaction between tourism enterprises and tourists through social media [8], etc. In-depth interview is usually used to excavate the perception of visitors about social media, and is used to research the influence of social media on consumer's decision-making [9]. It is worth noting that some researchers began to pay attention to how to effectively explore, obtain and scientifically analyze data through social media, and to use those data for tourism research and marketing. Many scholars have recognized the enormous potential of social media in the tourism marketing, but most of them remain their researches on quoting the western researches and describing the tourism format, lack of statistical data and in-depth quantitative analysis.

\section{Progress in Research and Application of Social Media in Tourism}

Research an Application on Tourists' Behavior. The application of social media enables people to share travel experiences, its content affects the tourist's planning and even their travel decisions [10]. The emergence of social networks has radically changed the way that tourists make a travel plan [11]. Due to potential visitors can make decisions based on the travel experience from others, it was found that social media has greatly affected the travel decision of tourists by electronic word-of-mouth [12]. Domestic scholars have realized social media has a strong appeal and influence for tourists [13], but the related research only remain in the observation and description of facts, lack of analysis and induction. Scholars in China have not yet analyzed the different types of user's preferences to social media because of their backgrounds, and have no attention to other aspects.

Research and Application of User Generated Content. From searching results of Chinese literatures, it is found that Chinese scholars tend to use network user generated content (UGC) as a tool, and use it for the destination management, such as studied tourist route planning based on UGC [14], discussed the relationship between UGC and destination image cognitive [7], and so on. Network travel information, especially the credibility, objectivity and authenticity of user's original content of the users, is very important. These contents are more credible than the official information, and 
gradually become an important source of information to potential visitors [15]. Users have different degrees of trust in different sources of network information. Some studies found that people believe that the most reliable source of information is the government's Websites, and professional travel sites are more credible than the comprehensive social media [16].

Application in Destination Management. Chinese scholars are enthusiastic about the use of social media for tourist destination marketing. Chinese literature about social media for tourism marketing occupies the largest proportion in the search results. At beginning of introducing the social media into tourism, some scholars have indicated that it can be used for destination marketing. Later, it is believed that will bring about the marketing change and innovation of tourist destination and tourism enterprises [17]. In addition, there are a lot of empirical researches on social media marketing for different levels (such as national, provincial and municipal enterprises and so on). Scholars also pay attention to the network word-of-mouth marketing, social media marketing costs and other issues.

Application in Tourism Crisis Management. The role of social media in tourism security is a new research topic. Micro-blog can play a certain role in tourism crisis management [18]. By studying on time management of tourism crisis, it is found that the use of social media in many countries is mainly related with accident, weather, political crisis and other factors. Tourism organizations should contact all stakeholders together through social media, and make crisis management [19]. Through the analysis of a large number of user generated content (UGC), someone found that social media have become a main way to show the tourist's complains [20]. Therefore, it is necessary to effectively manage social media, to reduce the negative effect on tourism enterprises.

Research and Application on Tourism Culture. Social media is changing the tourism culture. The traditional tourism culture is subversive, while the new tourism culture is forming. The intersection of huge tourists flow and modern social media can complete the creative construction and experience of cultural value for tourism heritage [21]. Modern tourists, especially those backpackers, have a highly dependence on high technology and communication tools in the whole process of traveling. Social media has an advantage on term of knowledge production ability and availability, so that it has made a great contribution in tourism knowledge sharing, creative inspiration and so on. Social media has become an important research tool in the field of tourism and hotel management [22]. Social media provides data, new research subjects, topics, and opportunities for many studies. At the same time, the data selection method, the protection of intellectual property rights and other important issues should be solved urgently in the process of study [11].

\section{Conclusions}

The continuous development of social media has a great impact on all aspects of tourism activities. For tourists, social media has changed the way to search, discover, read, and use travel information. For tourism enterprises, social media provides new management methods, marketing modes and business models. In the future to further develop the creative potential of social media, we should more efficiently utilize and share social media resources, while make a classification management to visitors, take timely interaction with them, make precision marketing, establish a positive image and reputation, and improve customer's loyalty and revisit rate.

Now the research contents are mainly focused on social media and tourist behavior, tourism information, tourism marketing, crisis management, tourism culture and so on. In the future, the scales of research should be further widened. The research should combine with the actual situation of local economy, culture and other issues. And the research efforts should be made on the aspects of community participation, integrated marketing tools, tourism destination marketing, and tourism law perfecting, etc.. Quantitative research should be used in the research. Non conventional sources of text on social media should also be included in our study. 


\section{Acknowledgements}

This work was financially supported by Social Science Foundation of Jilin Provincial Education Department ([2014] No.427) and the Research and Development Project of Jilin Engineering Normal University.

\section{References}

[1] P.W. Williams, K. Stewart and D. Larsen: Journal of Travel Research, Vol. 11 (2011) No.4, p.219-226.

[2] Information on https://en.wikipedia.org/wiki/Social_media\#cite_note-Buettner2016b-1

[3] Information on http://www.chyxx.com/industry/201605/415536.html

[4] J. Fotis, D. Buhalis and N. Rossides: Information and Communication Technologies in Tourism (Helsingborg, Swedish, 2012)

[5] X.L. Du: Science and Technique, Vol. 12 (2012) No.7, p.176-179.

[6] Y.X. Wang: Journal of Central South University of Forestry \& Technology (Social Sciences Edition), Vol. 7 (2013) No.5, p.22-31.

[7] S. Li: The Analysis of Attractiveness of the Travel Destination Based on UGC (MS., Harbin Institute of Technology, China 2011), p.23.

[8] H.F. Wu: The Study of the Text Production of Travel Guides in Tourism Websites: By Applying the Case Study of Mafengwo.com. (MS., Nanjing University, China 2013), p.17.

[9] M. Peng: Role of Micro- blogging in the Tourist Information Service: A Case Study of Tourist Destination Micro- blogging (MS., Anhui Normal University, China 2013), p.32.

[10] J. Yan, Y.B. Jiang and Y.P. Chang: Management Review, Vol. 23 (2011) No.12, p.84-91.

[11] Y.H. Huang: Tourism Tribune, Vol. 29 (2014) No.8, p.9-11.

[12] H. Li: Research on the Motivation of Internet Word-of-mouth Spread Behaviour of Tourist in Web 2.0 (MS., Hubei University, China 2013), p. 25.

[13]F. Zhang: Chinese tourists are enthusiastic about social media, China Tourism News, 2013-02-06.

[14] X. Lu: Travel Route Planning Based on User- generated Content (MS., Tianjin University, China 2013), p.23.

[15]L. Pan: The integrity research of UGC on travel booking website basing on interface design (MS., Shanghai Jiao Tong University, China 2011), p.14.

[16] L. Liu: Research on Personalized information service of Mobile Tourism (MS., Guangzhou University, China 2012), p.20.

[17] T. Zhao: Media Observation, Vol. 12 (2007) No.7, p.48-49.

[18] J. Luo. Tourism Overview, Vol. 2 (2012) No.9, p.39-40.

[19]Q.Chen: Research of tourism critical incident management-Take Sanya tourism crisis as an example (MS., Hunan Normal University, China 2013), p.45-47.

[20] Y. Liu and C.J. Li: Journal of Central South University of Forestry \& Technology (Social Sciences), Vol. 8 (2014) No.1, p.1-5+23.

[21]X. Wang, T.Q. Zou and W.H. Yang: Resources Science, Vol. 12 (2013) No.35, p.2352-2358.

[22] C.F. Liu: Spring airlines social media marketing strategy (MS., Donghua University, China 2014),p.67-68. 